\title{
The development of a web-based information tool for cross-border prevention and control of methicillin resistant Staphylococcus aureus
}

\author{
Fenne Verhoeven, ${ }^{1}$ Ron M.G. Hendrix, ${ }^{2}$ Inka Daniels-Haardt, ${ }^{3}$ \\ Alexander W. Friedrich, ${ }^{4}$ Michael F. Steehouder, ${ }^{1}$ Julia E.W.C. van Gemert-Pijnen ${ }^{1}$ \\ ${ }^{1}$ University of Twente, Netherlands; \\ ${ }^{2}$ Regional Public Health Laboratory Twente Achterhoek, Netherlands; \\ ${ }^{3}$ State Institute of Health and Labour (LIGA), Münster, Germany; \\ ${ }^{4}$ Institute of Hygiene, University Hospital Münster, Münster, Germany.
}

doi:10.3396/ijic.V4i1.006.08

\section{Introduction and background}

Increased international trade in health services can be an important cause of the rapid spread of health care associated infections, such as methicillin-resistant Staphylococcus aureus (MRSA). This particularly holds for European borders, where trans-border movement of health care workers (HCWs) and patients to supply and access medical services abroad heighten the threat of cross-border disease transmission, particularly when patients and HCWs are transferred from a middle- or high-endemic country, such as Germany, to a lowendemic country, such as the Netherlands, with an MRSA prevalence of $20 \%$ and $1 \%$, respectively. ${ }^{1,2}$ The occurrence of health care associated infections like MRSA is detrimental both to patients' and $\mathrm{HCWs}^{\prime}$ safety and, consequently, to quality of care. ${ }^{3}$

In order to ensure patient safety and to equalize quality of care in cross-border health care settings, prevention of health care associated infections need to be structurally addressed by synergic efforts of the health care actors representing the complete chain of care, on both sides of the border. An example of such an effort is EUREGIO MRSA-net Twente/Münsterland, in which local health care providers exchange knowledge and technology in order to reduce MRSA to acceptable levels both inside and outside the hospitals., ${ }^{4,5}$ Three years after its launch in 2005, the project has now established a cross-border infrastructure for a EU regional network of the major health care providers (e.g. hospitals, labs, public health services, GPs), a prevalence screening to determine regional risk factors, a concerted euregional on-admission screening scheme, and an online spa typing network for the early detection of special MRSA spa types. ${ }^{4}$ In addition, in order to build collective awareness for MRSA and health care associated infection in general, over $200 \mathrm{HCW}$ training courses were given, and education materials were developed to adequately inform the public. Moreover, since most important instruments needed to successfully implement infection control measures are guidelines and locally adapted protocols, both German and Dutch protocols have been optimized for cross-border collaboration on fighting MRSA. These

\section{Corresponding author}

Fenne Verhoeven, University of Twente, Netherlands 
protocols, complemented with practical information, are now accessible online through a web-based MRSA information tool (www.mrsa-net.nl).

The Internet has evolved as a potentially useful tool for protocol education, dissemination, and implementation because of its open standards and its ability to provide concise, relevant clinical information at the location and time of need. ${ }^{6}$ Previous research showed that $\mathrm{HCWs}^{\prime}$ compliance is better with electronic guidelines than with paper guidelines when tested in clinical scenarios. ${ }^{7}$ On top of the advantages the Internet has to offer, web-based tools have the potential to combine information to relevant activities by automation of the information seeking and retrieval processes to provide high-quality and appropriate answers to clinical questions. Web-based tools have been implemented for several disease models: asthma, ${ }^{6}$ low-back surgery, ${ }^{8}$ upper and lower digestive endoscopies, ${ }^{8}$ and also infectious diseases such as tuberculosis preventive therapy, ${ }^{6}$ and hepatitis $C^{9}$. However, to our knowledge, a web-based information tool aimed at the prevention and control or MRSA does not yet exist.

In order to ensure that our web-based information tool's functions optimally meet $\mathrm{HCWs}^{\prime}$ needs, we applied a user-centered design approach. ${ }^{10}$ This paper describes the steps that appeared to be crucial for the development of a tool that aims to provide HCWs with acceptable, applicable, user-friendly and high quality MRSA guidelines that enhance the decision making process to deliver safe health care.

\section{Methods \\ Respondents}

Study participants involved nurses and physicians from two Dutch and two German general hospitals in the EUREGIO Twente/Münsterland, varying in size from 314 to 1500 beds. A random sample involving HCWs from different types of wards with varying risk management problems was selected by the researcher (intensive care unit, surgery, internal medicine, and paediatric ward). All data were collected between March 2006 and September 2007. Table I shows the research methods that were deployed successively during the development process of the web-based MRSA information tool.

\section{Design team}

The web-based MRSA information tool was developed by the interdisciplinary project team of EUREGIO MRSA-net, comprising medical microbiologists, infection control nurses, physicians from municipal health services, behavioural scientists, application developers, and interface designers from both Germany and the Netherlands.

\section{Needs assessment}

We performed a needs assessment through a deep qualitative analysis of $\mathrm{HCWs}^{\prime}$ needs and their current activity and work habits to ensure that the tool can be successfully integrated in daily work practice. ${ }^{10}$ The needs analysis included three stages. We started with systematically analyzing which information is communicated in current Dutch and German national MRSA protocols, and how this information is presented. This systematic analysis provided us with a structure according to which we could present the information on our web-based tool. ${ }^{11}$ Subsequently, scenario testing was used among $28 \mathrm{HCWs}$ to identify user requirements for the web-based tool. Scenario testing involves users performing simulated tasks based on clearly defined scenarios. This technique has previously been established and used successfully to test clinical decision-making., ${ }^{72}$ By detecting and diagnosing problems that occurred with HCWs applying paper-based MRSA protocols in practice, we were able to determine the discrepancy between the desired and the perceived protocol information and the protocol's usability for HCWs. Finally, we completed the needs assessment with a survey among $217 \mathrm{HCWs}$ to determine how the web-based tool could best be implemented in practice. ${ }^{13}$

\section{Goal articulation}

The needs identified during the needs assessment phase were evaluated by the EUREGIO MRSA-net Twente/Münsterland project team, in order to select and prioritize those needs to be addressed. The issues taken in consideration included the objectives of the EUREGIO MRSA-net project, the consequences of the needs not being met, the available time, budget, and expertise for the EUREGIO-project, and the relation to other project activities. The resulting goals of the tool were articulated during a project discussion meeting. 


\section{Table I: Overview of the development process of the web-based MRSA information tool}

\begin{tabular}{ll} 
Instrument & Objective \\
\hline Document & Ensure that web-based tool \\
analysis & adequately presents useful, \\
& resource-rich protocol \\
& information to the intended users
\end{tabular}

\section{Procedure}

Based on literature, a framework for cross-border guidelines was developed. Two researchers independently coded national Dutch and German guidelines according framework. Results were compared and consensus about coding differences was reached by discussion.

\begin{tabular}{ll}
\hline Scenario & Explore usability, acceptability \\
testing & and applicability problems that \\
& occur with paper-based protocol \\
& use, and thereby identification \\
& of user needs.
\end{tabular}

Respondents ( $\mathrm{n}=28$ ) were asked to verbalize thoughts and actions (think aloud) while using the protocol to solve an MRSA-related problem: e.g. "You are attending to an MRSA patient in an isolation room. Using the protocol, can you say aloud which safety preventive measures you must take?"

\begin{tabular}{ll}
\hline Survey & $\begin{array}{l}\text { Determine how the web-based tool } \\
\text { can best be implemented in clinical } \\
\text { practice }\end{array}$
\end{tabular}

Confidential, self-administered close-ended questionnaires (based on existing instruments fitting the study themes) with return envelopes were distributed to a random sample of $320 \mathrm{HCWs}$. After check for completeness, final sample size was $\mathrm{n}=217$. All data were processed in SPSS 12.0. Structural Equation Modeling techniques (AMOS 5 Software) were used to identify intervention variables.

\begin{tabular}{ll}
\hline Screen & Validate user requirements and \\
mock-ups with & allowance of users to contribute \\
semi-structured & to web-based tool's design \\
interviews &
\end{tabular}
While being shown the mock-up prototype on a laptop, participants $(n=14)$ commented on the look-and-feel, expectations, and usability aspects such as credibility, lay-out, comprehensiveness, etc. Mock-up prototypes each consisted of a homeand a search page. Comments were categorized and quantified. Most frequently mentioned comments were taken into account for design revisions of the web-based tool.

\begin{tabular}{ll}
\hline Card Sort & Design information structure \\
Study & of web-based tool
\end{tabular}

Participants ( $n=10$ ) sorted cards with content items (questions) for the web-based tool, and labelled the groups created. Hand sorted card data were entered into WebSortTM tool, and analyzed with IBM's EZ sort application, which visualizes the differences and similarities between items in a tree-diagram based on cluster analyses. Besides, a qualitative analysis of the labels and the interviews was conducted. 
Subsequently, we determined based on both literature and experience, what HCWs need to know or be able to do to achieve these goals, which resulted in the web-based tool's functional requirements. The tool's goals and functional requirements are shown in Table II and III of the results section, respectively.

\section{Design and development}

Once the goals and requirements were understood, the actual design process started. We attempted to visualize the web-based tool by creating mock-up prototypes and developed a blueprint for the webbased tool. Two mock-up prototypes of the homepage and the search page were created, based on common guidelines for web design and usability. ${ }^{14}$ We evaluated the prototypes by interviewing 14 intended users. Users are in a unique position to provide early, authentic feedback. They know what they need and want and can respond to the design.
The structure of the information provided by our web-based tool was a design challenge of particular concern. To ensure a practical use of the tool by HCWs, the tool had to present very complex actionoriented information in a clear and simple a manner, with a very easy navigational structure. To guarantee this, we applied a Card Sort Study among ten HCWs. Card sorting is often applied as a user-centered method for designing the information structure of a web site. ${ }^{15}$ The initial scenario testing (see above) had provided us with 165 key questions that were unanswered by the current MRSA protocols, while their answers are nevertheless required for HCWs to deliver safe health care. During the Card Sort Study, ten respondents sorted these 165 questions into meaningful groups that eventually served as the web-based tool's navigation structure.

Table II. Identified goals of the web-based MRSA information tool based on needs assessment

\begin{tabular}{|c|c|}
\hline Method & Goals \\
\hline $\begin{array}{l}\text { Document } \\
\text { review }\end{array}$ & $\begin{array}{l}\text { - The web-based tool should integrate both (1) the approach that views an MRSA protocol } \\
\text { solely as documentation of the health and safety policy of the hospital (Germany) and (2) } \\
\text { the consideration of MRSA as a collective management problem (the Netherlands). This can } \\
\text { be realized by stressing that a successful MRSA prevention and control policy requires the } \\
\text { (cross-national) participation of all HCWs within and between institutions because deviant } \\
\text { behaviour from just one HCW may lead to an outbreak, and giving concrete tools how to } \\
\text { perform this in practice. Adding items such as risk categories and decision trees simplify the } \\
\text { decision process for an individual HCW and thereby possibly decrease the numerous } \\
\text { possibilities for an HCW to apply knowledge inadequately and make mistakes. }\end{array}$ \\
\hline Scenario testing & $\begin{array}{l}\text { - The web-based tool should enable HCWs to find answers to all their practical and } \\
\text { medical questions concerning MRSA and allow them to take adequate decisions for daily } \\
\text { - Thork practice in order to prevent failures. } \\
\text { inadequate decisions and eventually in unsafe health care, by providing HCWs with } \\
\text { acceptable, applicable, and user-friendly guidelines. } \\
\text { - The web-based tool should focus on specific clinical circumstances rather than general } \\
\text { situations, and employ a question-oriented approach, and take into account different } \\
\text { search strategies that HCWs apply. }\end{array}$ \\
\hline Survey & $\begin{array}{l}\text { - The reinforcing factor 'safety climate' is the most important factor for successful } \\
\text { implementation of the web-based tool, in order to create a collective awareness among } \\
\text { health care workers to avoid spread of MRSA. }\end{array}$ \\
\hline
\end{tabular}




\section{Results}

In this section, the different steps of the web-based tool's development process will be successively presented. First, the problems recognized during the needs assessment will be elucidated. Based on the needs assessment, the tool's goals were formulated which led to the tool's functional requirements, which can be found in Table II and III, respectively. Eventually, we took into account these functional requirements when designing the actual tool, which we present in Figure 3 and 4 . Before the actual launch of the webbased tool, we validated the tool's design and content among the intended users.

\section{Needs assessment}

a. Document analysis to ensure that web-based tool adequately presents useful, resource-rich protocol information to the intended users.

\section{Table III. Functional requirements generated for the web-based MRSA information tool based on needs assessment}

\begin{tabular}{|c|c|}
\hline Method & Goals \\
\hline Document & $\begin{array}{l}\text { - Guidelines should be presented according to a structured format and integrate two visions: } \\
\text { (1) the approach that views an MRSA protocol solely as documentation of the health and } \\
\text { safety policy of the hospital, and (2) the consideration of MRSA as a collective management } \\
\text { problem: } \\
\text { - Title } \\
\text { - Short answer: Information and communication-oriented (cf. 2): Explicitly defining tasks } \\
\text { and responsibilities. } \\
\text { - Comments: User-centered and risk-focused (cf. 2): Non-textual elements such as decision- } \\
\text { trees that tell the user what should be done, and stressing the risk consequences of non- } \\
\text { compliance with the protocol. } \\
\text { - Sources: Evidence-based and focus on legislative regulations (cf. 1): Referral to law or } \\
\text { evidence in literature } \\
\text { - Multimedia examples: User-centered (cf. 2): E.g., an instruction movie or pictures of how } \\
\text { to adequately apply safe work practices). }\end{array}$ \\
\hline Scenario testing & $\begin{array}{l}\text { - Guidelines should prevent information and applicability problems. Therefore, the } \\
\text { following features should be included in the web-based tool: } \\
\text { - Search engine: enables HCWs to pose a question in their own language/ vocabulary. } \\
\text { - Menu structure: where the } 165 \text { questions that resulted from the scenario testing are } \\
\text { categorized into logical groups, according to the target group. } \\
\text { - Frequently asked questions. } \\
\text { - Breadcrumb trail to help users keep track of their location within the system. } \\
\text { - Structured answer; for ease of comprehension, each of the } 165 \text { answers are structured in } \\
\text { the same way, with most important items placed toward the top of the page. }\end{array}$ \\
\hline Survey & $\begin{array}{l}\text { Interventions should be particularly targeted at creating a strong safety climate in order to } \\
\text { create collective awareness among all HCWs about protocol compliance, based on } \\
\text { organizational behavioural management, characterized by: } \\
\text { - focus on current determinants of behavior, not prior history } \\
\text { - emphasis on overt behavior change as the criteria for treatment evaluation } \\
\text { - careful targeting of critical behaviors } \\
\text { - emphasis on measuring behaviors and monitoring their outcomes } \\
\text { - emphasis on the involvement of all staff in its development and application }\end{array}$ \\
\hline
\end{tabular}


We systematically analyzed existing Dutch and German national MRSA protocols, in order to determine how the information in our web-based tool could best be presented to the target group. Two approaches for structuring MRSA protocols emerged from this analysis: (1) Considering a protocolasadocumentcontainingsafetyregulations by stressing legislative regulations and evidence, as is the case in Germany, and (2) approaching the control of MRSA as a collective management problem, by stressing risks, information- and communication- items, which occurs in Dutch protocols. We integrated these two approaches in our web-based tool by presenting the information as such that both safety regulations and tangible information and communication aspects are present, complemented with practical information from everyday work practice (see Table II and III). By including both approaches, we strived to enhance that by using the web-based tool, HCWs become aware of their role in a large health care network in which they have to interact in order to prevent and control MRSA. This is particularly relevant in cross-border health care settings, where HCWs from different institutions in various countries have to cooperate.

b. Scenario tests to explore usability problems that occur with paper-based protocol use, and identification of user needs

HCWs from both countries encountered several problems while using the protocol because protocols required them to apply knowledge outside their current realm. $49 \%$ of scenarios that respondents were asked to solve by means of the paper-based protocol, could not be completed successfully, due to the following causes:

1. Incomprehensive information ( $40 \%$ of cases): For instance, the protocol did make clear which protective clothing should be worn when entering an isolation room, but it did not tell to respondents in which order the clothing should be taken on and off.

2. Information retrieval problems (23\%): Respondents could not find relevant information because of the size of the protocols and a discrepancy between the search terms used by HCWs (e.g., "treatment") and the vocabulary applied in the protocol (e.g., "decontamination procedure").

3. Inapplicability of information (20\%): Outdated information, and a discrepancy between the protocol's rules and the possibilities of executing them in practice. E.g., the protocol requires each (suspicious) MRSA-positive patient to be treated in preventive isolation, while on particular wards no isolation facilities are available.

4. Incomprehensible information (15\%): Most HCWs appeared to be unfamiliar with the professional jargon used in the protocols.

While German HCWs particularly came across incomprehensive information, Dutch HCWs struggled with the applicability of the information. In total, 165 key questions relating to everyday work practice were found, for which the protocol did not provide an adequate answer. Because we strived with the web-based tool to provide HCWs with acceptable, applicable, user-friendly protocols that enable them to deliver safe health care, we included the answers of these 165 questions in the tool's content. In order to prevent usability problems that occurred with paper-based protocol use, the answers to the questions were presented according to the structure that appeared from the document analysis. Furthermore, the tool had to include features such as a search engine, usercentered navigation structure and a breadcrumb trail (see Table III).

c. Survey to determine how web-based tool can best be implemented in practice.

The survey results showed that in order to enhance successful implementation of the web-based tool, the tool should be embedded in the hospital's safety climate. Respondents indicated that in a firm safety climate, risks, health benefits, and dangers of multi-resistant organisms are explicitly discussed. Therefore, the respondents deemed it important that these risk elements are also actively advocated on the web-based tool in order to increase HCW's awareness of the risk to acquire or transmit MRSA in case of non-compliance with the protocol. 


\section{Goal articulation}

Potential solutions to the problems recognized by the needs assessment were identified and project goals were drafted by the project team of EUREGIO MRSAnet Twente/Münsterland to describe what users should be able to do as a function of using the web-based tool (see Table II). Goals were then broken down into the tool's functional requirements. Table III shows a list of the required characteristics.

\section{Design and development}

a. Screen mock-up interviews to validate user requirements and allow of users to contribute to web-based tool's design

We applied research-based web design and usability guidelines when creating two nonworking mock-up prototypes, taking into account the needs assessment results. ${ }^{15}$ The mockup prototypes each consisted of two pages (a homepage and a search page, see Figure 1 and 2), and showed the system's lay-out and navigation.
When asked to indicate their preference for one of the two prototypes, 12 out of 14 participants chose prototype 1 , mainly because of "the convenient structure" $(n=3)$, "the topic MRSA is presented clearly" $(n=4)$, and "the trustworthiness suggested by the logos of the involved organisations $(n=2)$. Prototype 2 was evaluated as "unattractive" ( $n=10)$, "too commercial $(n=7)$ ", and "inconveniently arranged" $(n=6)$. Hence it was decided that the final lay-out of our web-based tool should be based on prototype 1 .

b. Card Sort Study to design information structure of web-based tool

The Card Sort Study resulted in ten categories according to which the 165 questions that resulted from the scenario testing can be structured. The categories, each with an example to illustrate the practical approach of the web-based tool, are presented in Table IV. Compared to the general tables of content of MRSA protocols, it is

\section{Table IV. Categories resulting from the Card Sort Study, with an exemplary question}

\section{Category Question example}

1. MRSA in general Does MRSA threaten the future?

2. Screening Sometimes, screening cultures are taken among HCWs in case of an MRSA-patient, but sometimes not. Why is there no consequent screening policy?

3. Protective measures In which order should I put on and off personal protective equipment?

4. Disinfection measures How often should I disinfect medical equipment and medical instruments that are used in an isolation room?

5. Admission Which measures should I take when an MRSA-positive patients enters the emergency room?

6. Transport An MRSA-positive patient should be transported from one ward to another within the hospital. Which personal protective equipment should I wear when accompanying this patient?

\begin{tabular}{|c|c|}
\hline 7. Discharge & $\begin{array}{l}\text { Which materials may I throw away after discharge of an MRSA-positive patient } \\
\text { from an isolation room? }\end{array}$ \\
\hline 8. Treatment & What are the side-effects of the eradication-therapy? \\
\hline $\begin{array}{l}\text { 9. MRSA and } \\
\text { your own health }\end{array}$ & Am I allowed to receive visitors at home, when I am MRSA-colonized? \\
\hline 10. Communication & $\begin{array}{l}\text { There is an MRSA-outbreak at my ward. Who should I inform about this and wh } \\
\text { should I tell? }\end{array}$ \\
\hline
\end{tabular}


remarkable that the search structure based on the Card Sort Study includes a category on "MRSA and your own health", and a category on "Communication". We also found that the categories can best be ordered according to the stages of the patient's presence in the hospital: "Admission", "Transport" and "Discharge". It thus appeared that HCWs desired a more actionand communication-oriented way of structuring information than is the case in current Dutch and German MRSA protocols.

\section{System description of the final web-based MRSA information tool}

The results from each of the five studies conducted during the development process were integrated in a final version of the web-based MRSA information tool. Figure 3 and 4 show the final version of the homepage and an answer page. The 165 questions that emerged from the scenario testing served as input for the tool's content and were answered based on national MRSA guidelines, and complemented by the Dutch and German medical microbiologists involved in the research project. Figure 4 shows how we took into account the different search strategies that users apply by facilitating the web-based tool with three different search options. The answers were directly communicated to the user in an instructional, imperative style. The users were addressed directly, e.g., "You first have to put on gloves, followed by a gown, etc." The answers were structured according to usability guidelines, with important items placed consistently at the top center, scroll stoppers were avoided as much as possible, moderate white spaces were used, and feedback was provided on users' location on the website by means of a breadcrumb trail on top of the page, to enable them to keep track of their location within the system. ${ }^{14}$

Users were allowed to decide upon the level of detail of the answer they wish to obtain, because next to the short answer, in-depth elaborations were provided, even as sources (e.g., scientific literature, newspaper articles, links to other websites for further reading and explanations of the suggested topics). If possible, multimedia examples (e.g., instruction movies, pictures of how to adequately apply personal protective equipment) were added as well. Furthermore, a specific machine learning functionality was incorporated in the web-based tool. This function enabled that the questions that have been looked up most frequently, are ranked higher in the list when the users user the search engine or browse through the categories.

A dedicated content management system enables the project team to add and delete questions to the web-based tool at any time and location. This system allows also to continually update the tool's content, which is of vital importance because infection control policies are frequently subject to changes. The content management system also allows the project team to access the logfiles that record all activity that occur on the web-based tool. The logfiles record standard data such as page views, session duration, IP addresses, results of the feedback function, and also the queries users entered in the search engine. Questions that are
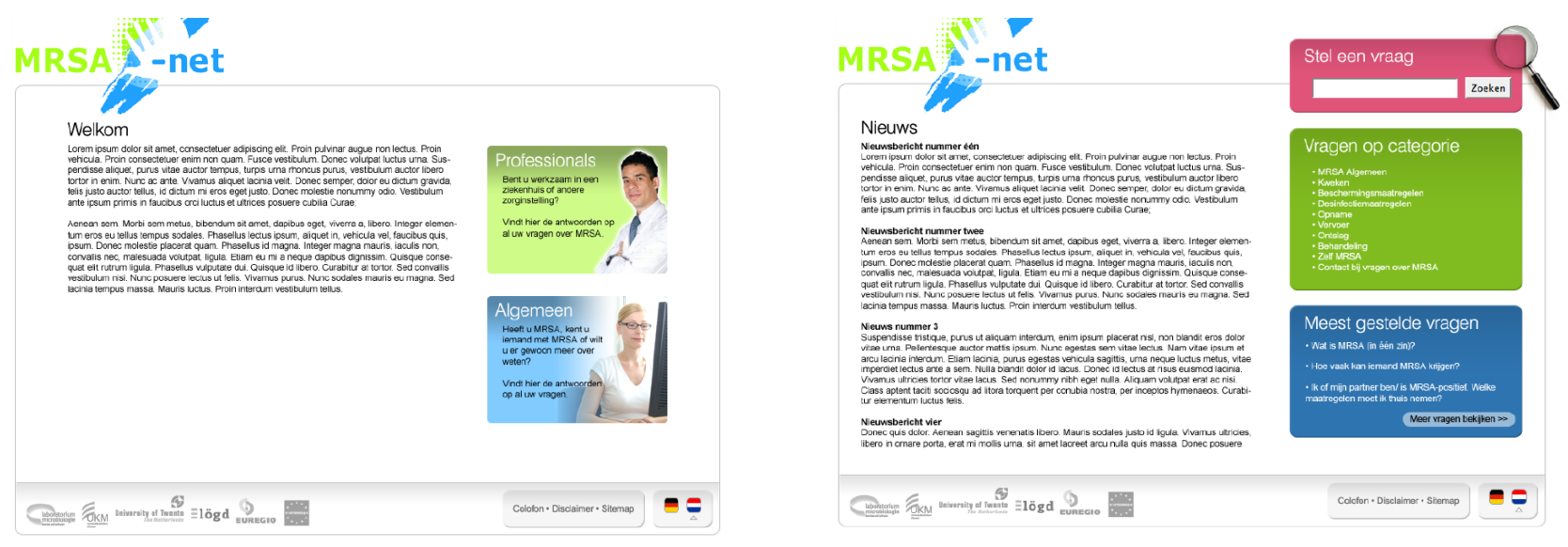

Figure 1. Screen mock-ups prototype 1: Homepage on the left, search page on the right 

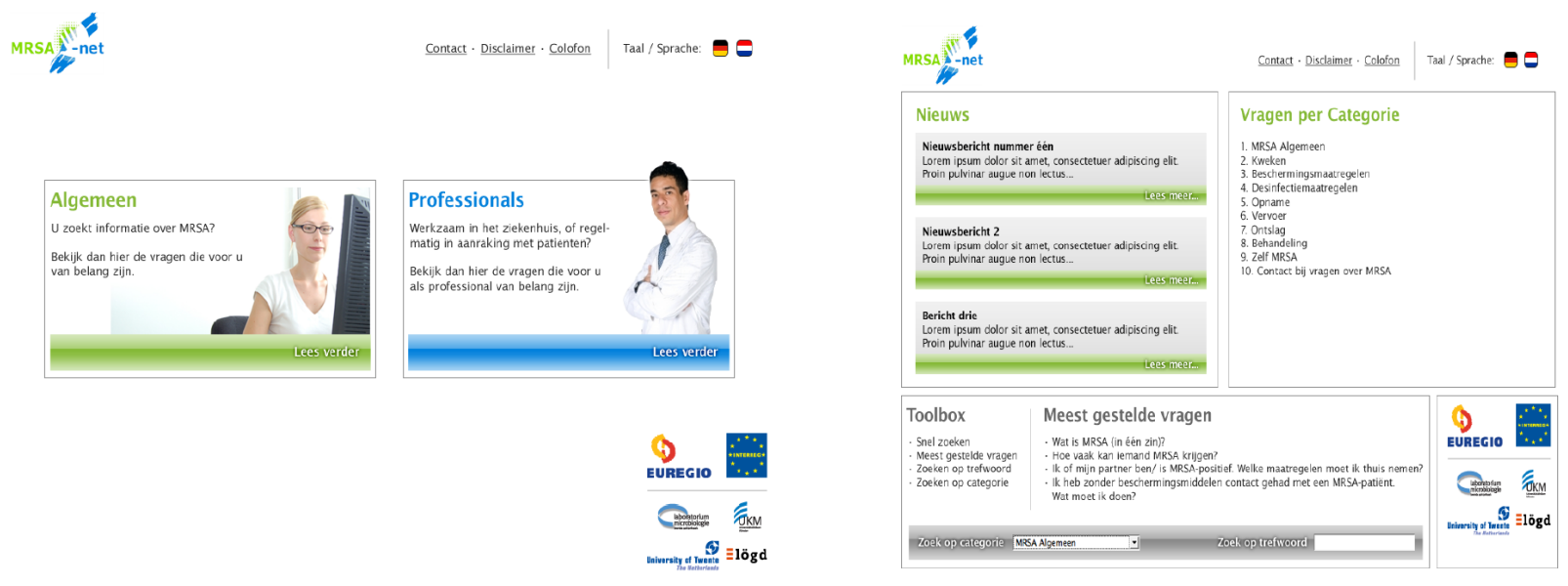

Figure 2. Screen mock-ups prototype 2: Homepage on the left, search page on the right

not answered yet by the web-based tool can be easily identified by the logfiles, and the project team can immediately add the question and its answer to the web-based tool, via the content management system.

\section{Discussion}

This study provided us with a web-based MRSA information tool, which was launched in February 2008 (www.mrsa-net.nl). The user-centered design approach described in this paper combined data of different data sources (during needs assessment, goal articulation, design, and development). This approach proved effective for enhancing users' involvement in the development process, which is often neglected in protocol development. ${ }^{12}$ Participatory development is important to create ownership and to foster the applicability of the protocol. The involvement of hospital management, users and other stakeholders is paramount for the eventual compliance with guidelines. ${ }^{16}$

The Internet is increasingly facilitating communication in infectious disease surveillance and outbreak investigation, and can also prove to be an effective tool to disseminate clinical practice guidelines. ${ }^{17,18}$. Online guidelines offer the potential for rapid up-dating, and they allow for the use of multimedia resources and hyperlinks ${ }^{6}$. In our study, the Internet enabled us to communicate guidelines to different occupational groups, taking into account different levels of detail various occupational groups are interested in, and different search strategies users maintain. In this way, the information has the maximum impact on knowledge, attitudes, and subsequent safety behaviour. ${ }^{19,20}$ Other features of web-based clinical guidelines systems are the potential for effective dissemination and free availability at the location and time of need. ${ }^{6}$ Because participants in our study indicated that they had a rather practical information need, we try to couple information in the web-based tool to relevant activity. By ensuring that the information is there when, where and how HCWs want it, we expect that good quality information forms the basis for HCWs to make informed choices about their actions. Because our tool is web-based, and computer and Internet facilities are available at practically every hospital ward nowadays, the web-based tool can be incorporated in the workflow of clinical care. Behavioural change occurs most effectively when coupled to relevant activity. ${ }^{6}$

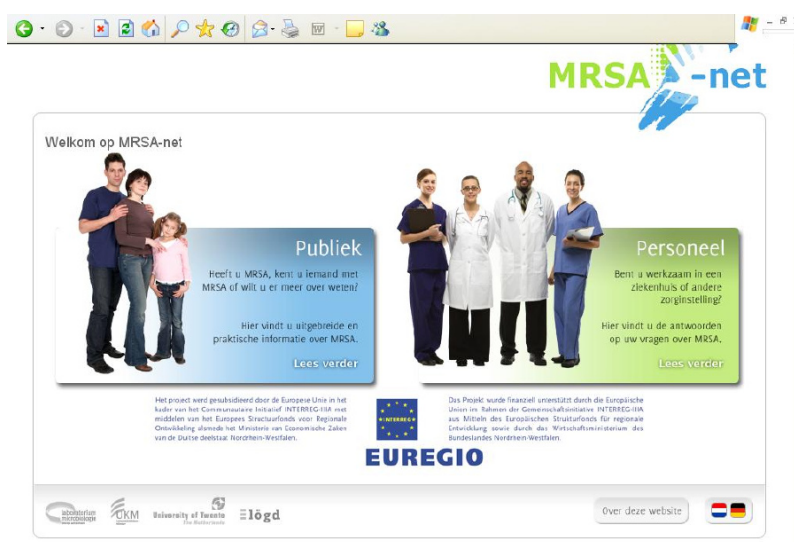

\footnotetext{
Figure 3. Final version of the web-based MRSA information tool's homepage
} 
Compliance with MRSA protocols is of vital importance, because the occurrence of multi-resistant microorganisms is detrimental both to the patient and HCWs' safety and, consequently, to quality of care. ${ }^{21}$ Previous research showed that computerbased guideline systems have been shown to improve prescribing practices, reduce serious medication errors, enhance the delivery of preventive care services, and improve adherence to recommended care standards. ${ }^{22}$ In future research, we will investigate whether the web-based tool will contribute to a higher compliance with MRSA guidelines and eventually a reduction in MRSA rates.

By considering that a successful MRSA prevention and control policy requires the (cross-national) participation of all HCWs within and between institutions because deviant behavior from just one HCW may lead to an outbreak, and giving concrete tools how to perform this in practice, our web-based tool can create a form of awareness amongst HCWs which is needed in our era of increasing international trade in health services.

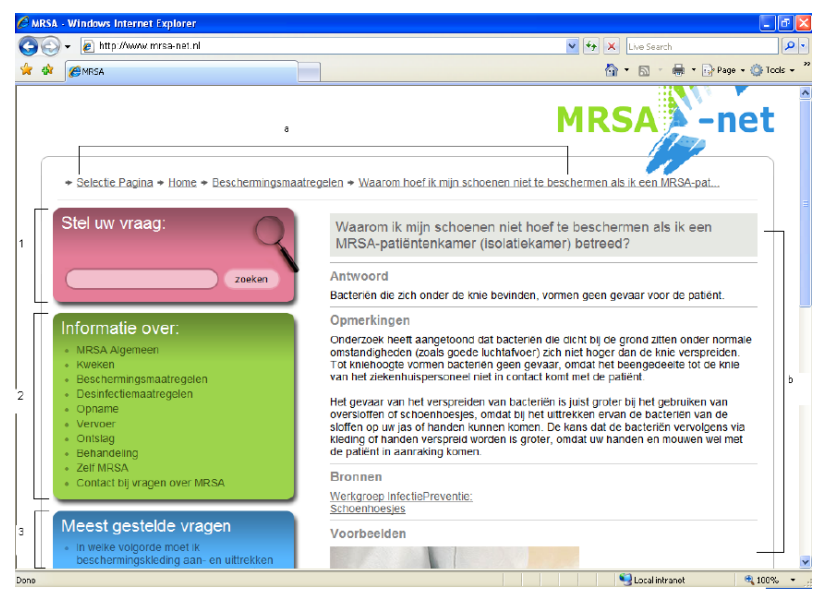

Figure 4. Final version of a web-based MRSA information tool's answer page. $1=$ search engine; 2=Categories resulted from Card Sort Study; $3=$ Most frequently asked questions; $\mathrm{a}=\mathrm{Breadcrumb}$ trail; $\mathrm{b}=$ Structured answer (title, short answer, comments, sources, multimedia examples, and related answers [not visible])

\section{Limitations}

Solely developing a web-based tool providing userfriendly infection control guidelines is not enough to enhance compliance with guidelines and eventually improve quality of care. For this, it is of vital importance to disseminate and implement the webbased tool successfully in the health care setting. Organisational and managerial support as well as systems for audit and evaluation are key elements in the successful implementation of guidelines in practice within the medical profession. ${ }^{23}$ But also the fact that the tool is web-based might cause implementation problems, such as HCW reluctance, proprietary interests, technical limitations, and local practice environments. The main limitations of the Internet include technical aspects imposed by the open standards and the challenge of controlling information quality. ${ }^{6}$ These limitations, however, do not necessarily impose disadvantages. The open standards available on the Internet allowed us to simplify our user interface within existing standards and thus ensure widespread availability and ease of navigation. To target the largest possible audience, and to achieve a high degree of evidence, we used nationally developed and widely applied clinical guidelines.

\section{Future research}

Larger studies are required to investigate the longterm impact of the web-based tool on compliance with safe work practices. Therefore, from February $19^{\text {th }} 2008$, when the web-based tool was officially launched online (see www.mrsa-net.nl), logfiles and an online survey based on existing instruments to measure usability, ${ }^{24}$ have been conducted in order to test the system's usability and see the web statistics to verify whether users have actually adopted the webbased tool in daily work practice. If necessary, the goals and functional requirements can be adapted according to the iterative nature of the design model. The evaluation results will be used for a future webportal on cross-border patient safety which will be more and more important as cross-border patient care and battle against antimicrobial resistance is an increasing European issue.

Furthermore, as MRSA is becoming ever moreprevalent outside the hospital, we also developed a web-based 
tool for patients and the general public according to the same user-centered design methodology. This webbased tool can empower consumers of health services, enabling them to make informed choices. ${ }^{25}$

\section{Acknowledgements}

This study was financially supported within the EUREGIO MRSA-net Twente/Münsterland under the European Regional Development Fund (ERDF) funded by the European Union within the Community Initiative INTERREG-IIIA (2-EUR-V-1=96) as well as by the Ministry of Economics from the German state of Nordrhein-Westfalen.

\section{References}

1. Pittet D, Allegranzi B, Sax $\mathrm{H}$, et al. Considerations for a WHO European strategy on health-care-associated infection, surveillance and control. Lancet Infect Dis 2005; 5: 242-250.

2. Deurenberg RH, Vink C, Oudhuis GJ, et al. Different clonal complexes of Methicillin-Resistant Staphylococcus aureus are disseminated in the EUREGIO Meuse-Rhine region. Antimicrob Agents Chemother 2005; 10: 4263-4271.

3. Pittet D, Donaldson L. Challenging the world: patient safety and health care-associated infection. Int I Qual Health Care 2006; 1: 4-8.

4. Friedrich, AW, Daniels-Haardt I, Gemert-Pijnen JEWC van, et al. Ein regionales Netzwerk zur Prävention und Kontrolle von Infektionen durch MRSA: EUREGIO MRSA-net Twente/ Münsterland. Epidemiologisches Bulletin 2007; 33; 307-311

5. Friedrich AW, Daniels-Haardt I, Sonntag AK, et al. Euregio MRSA-net Twente/ Münsterland. Westfälisches Arzteblatt 2006; 9; 51-52.

6. Thomas K, Dayton SC, Peterson MW. Evaluation of InternetBased Clinical Decision Support Systems. J Med Internet Res 1999; 1: e6.

7. Kolbe J, Vamos M, James F, Elkind G, Garrett J. Assessment of Practical Knowledge of Self-Management of Acute Asthma. Chest 1996; 109: 86-90.

8. Jeannot J, Scherer F, Pittet V, Burnand B, Vader J, Use of the World Wide Web to Implement Clinical Practice Guidelines: A Feasibility Study. J Med Internet Res 2003; 5: e12

9. Hedin K, Babic A, Frydén A. Take care: guidelines for patients with chronic hepatitis C. Stud Health Technol Inform 1999; 68: 783-788.

10. Kinzie $M$, Cohn W, Julian $M$ et al. A user-centred model of website design: needs assessment, user interface design and rapid prototyping. I Am Med Inform Assoc 2002; 9: 320-330.
11. Verhoeven F, Gemert-Pijnen JEWC van, Friedrich AW, DanielsHaardt I, Hendrix MGR. Euregional MRSA-prevention and control: A comparison of Dutch and German guidelines. Infectieziektenbulletin 2007; 4: 125-129.

12. Gemert-Pijnen J van, Hendrix MGR, Palen J van der, Schellens PJ. Performance of methicillin-resistant Staphylococcus aureus protocols in Dutch hospitals. Am I Infect Control 2005; 33: 377-384.

13. Dejoy DM, Searcy C, Murphy LR, Gershon RM. Behavioraldiagnostic analysis of compliance with universal precautions among nurses. J Occup Health Psychol 2000; 5: 127-141.

14. Koyani SJ, Bailey RW, Nall JR. Research-Based Web Design \& Usability Guidelines. Washington DC: U.S. Government Printing Office 2006.

15. Tullis, TS. Using Card-sorting Techniques to Organize your Intranet. Intranet Journal of Strategy \& Management 2003; 1: 1-9.

16. McCoy KD, Beekmann SE, Ferguson KJ, et al. Monitoring adherence to standard precautions. Am I Infect Control 2001; 29: 24-31.

17. Mikanatha NM, Welliver DP, Rohn DD, Julian KG, Lautenbach E. Use of the web by state and territorial health departments to promote infectious disease reporting [research letter]. J Am Med Inform Assoc 2004; 29: 1069-1070.

18. Ambresin AS, Acremont $\bigvee D^{\prime}$, Mueller Y, Martin O, Burnand B, Genton B. www.fevertravel.ch: An online study prototype to evaluate the safety and feasibility of computerized guidelines for fever in returning travellers and migrants. Comput Methods Programs Biomed 2007; 85: 19-31.

19. Sinkowitz-Cochran RL, Stein GP, Keyserling HL, Levine GL, Jarvis W, The Pediatric Prevention Network. The Internet: A practical example of the use of new technology in the assessment of vancomycin use in pediatrics. Am / Infect Control 2000; 28: 459-464.

20. Madle G, Kostkova P, Mani-Saada J, Weinberg J, Williams P. Changing public attitudes to antibiotic prescribing: can the Internet help? Informatics in Primary Care 2004; 12: 19-26.

21. Pittet $D$. Infection control and quality health care in the new millennium. Am J Infect Control 2005; 33: 258-267.

22. Kawamoto K, Houlihan CA, Balas EA, Lobach DF. Improving clinical practice using clinical decision support systems: a systematic review of trials to identify features critical to success. BMJ 2005; 330: 765.

23. Grimshaw JM, Russell IT. Effect of clinical guidelines on medical practice: a systematic review of rigorous evaluations. Lancet 1993; 342: 1317-1322.

24. Muylle S, Moenaert R, Despontin M. The conceptualization and empirical validation of web site user satisfaction. Information \& Management 2004; 41: 543-560.

25. Woolf S, Grol R, Hutchinson A, Eccles M, Grimshaw J. The potential benefits, limitations and harms of clinical guidelines. In: Eccles, M., Grimshaw, J, Eds. Clinical Guidelines. From Conception to Use. Radcliffe Medical Press Ltd.: Oxon 2000; 19-29. 